Julio Calvo Pérez

Universidad de Valencia

\title{
LA ORACIÓN DE RELATIVO EN QUECHUA: LA APORTACIÓN DE LOS MISIONEROS LINGÜISTAS
}

\section{La aportación de los misioneros y su proyección futura}

La primera gramática quechua conocida (Santo Thomas, 1560) no plantea de modo directo el problema de la oración de relativo, inexistente nexualmente en quechua. Resulta lógico que así sea en una primera gramática introductoria de una lengua y en un tiempo en que la sintaxis se centra, casi exclusivamente, en la concordancia y en el orden de las palabras. Sólo cabe encontrar ejemplos, en cuya descripción sea posible hallar claves para el análisis de tal construcción. Los rastros encontrados son:

1) La presencia de ciertos nombres verbales (Cap. VII, ff. 42-43), en que tenemos la frase Sullull ñini, suc sapalla Dios, hanampachap tukuy ima ayca cacpa, yachachic ruraquenc 'Digo y confiesso que es verdad que ay vn solo Dios criador y hazedor del cielo, y de la tierra, y de todo lo demás'. En ella, los adjetivos verbales yachakiq 'el que enseña' y ruraqin (hoy ruraqmi) 'el que hace' parecen hallarse en aposición al sustantivo principal Dios (hoy Diyus). Es la fórmula del español actual Dios [el] Enseñador, Dios [el] Hacedor, fórmulas sintéticas de Dios [el] que enseña y Dios [el] que hace, en fase previa a la construcción oracional plena Dios enseña, Dios hace.

2) El análisis del participio verbal perifrástico (Cap. VIII, ff. 43-47), en que nos encontramos con construcciones del tipo micoc cangui 'tú comes', «elegante manera de hablar» equiparable a la del' latín sum comedens, es legens. Domingo de Santo Thomas -ST a partir de ahora- asimila esta forma a la sintética micuni 'yo como', dado que el verbo en su enunciación sintética tiene dos significados: tanto la forma habitual 'suelo comer' como la resultativa 'he comido', perteneciendo la equivalencia lógicamente a la habitualidad que se desprende de la forma analítica micoc cangui (hoy mikhuq kanki) 'tú eres [el] comedor / comiente' o bien 'tú eres el que come'. Tampoco aquí se aclara si estamos ante una aposición o no, ya que ST destaca que es opcional el uso de la partícula de ornato me o mi: micocmi cangui, que haría del conjunto una forma bimembre de carácter atributivo, lo que en español se muestra mediante el artículo que sustantiva el adjetivo adjunto: tú eres el que come.

3) El recurso a la aproximación sustantivo / adjetivo (f. 45v): payconap coyascam 'cosa amada de ellos', cuyo análisis, tras la modernización ortográfica pertinente, es el que sigue:

(1)

$\begin{array}{llllll}\text { Pay- } & -k u n a & -q & \text { khuya- } & -s q a & -n \\ \text { pron3 }^{\text {a }} & \text { PL } & \text { GEN } & \text { amar } & \text { PPAS } & \text { VAL }\end{array}$

'De ellos en verdad [lo] amado'

Se trata de una construcción exocéntrica de genitivo, equivalente a un adjetivo analítico, pero que no llega a ser todavía tal adjetivo y menos una forma analítica de nivel superior: una oración de relativo. 
4) El desarrollo de los pronombres relativos (Cap. 15, f. 54). Pese a la coincidencia de nombre, el relativo de ST no es exactamente el que pretendemos desarrollar aquí, ya que se inclina directamente por las adjunciones apositivas de carácter anafórico. El autor dice de los relativos «que hazen relación de lo passado» y que son los cuatro pronombres primitivos de tercera persona siguientes: pay 'él/ella', chay 'ese', chaqay 'aquél' y kikin 'mismo'. Por ejemplo: Pedro callpanc, paypas mana çamancchu 'Pedro corre, el cual no está descansando', cuya versión literal sería: 'Pedro se esfuerza; él, en primer lugar, no descansa'. Habría que observar la dificultad de presentar estas formas en primera o segunda persona, pese a la presencia de kikin:

(2)

$\begin{array}{llllllll}\tilde{N} \text { uqa } & \text { mikhu- } & n i, & k i k i- & -y & -p a s & \text { upya- } & n i \\ \text { yo } & \text { comer } & 1^{\text {a }} & \text { mismo } & 1^{\text {a }} & \text { ADIT } & \text { beber } & 1^{\text {at }}\end{array}$ 'Yo como <habitualmente>; yo mismo, en primer lugar, bebo <habitualmente>'

cuya versión en relativo moderno en español sería: 'Yo, que sobre todo bebo, habitualmente como'.

5) El uso esporádico esclarecedor (Cap. 24, f. 72). Al hablar de la figura de la evocatio (vocativo), ST vuelve al estilo de sus frases favoritas: Noca, Domingo, Dios hananc pachap cay pachap yachachicta riccini 'Yo, Domingo, conozco a Dios, que crió el cielo y la tierra'. La oración fuera del vocativo Domingo aporta un OD complejo con el acusativo -ta, en que no hay aposición puesto que tal objeto no repite la terminación de caso en la palabra Dios: Diosta. Aquí no cabría traducir como '...a Dios, al que crió...', sino que la adjunción de un adjetivo verbal al sustantivo constituye un bloque único:

(3)

Diyus hanan pacha- -q kay pacha- -q yacha- -chi $-q \quad-t a$

Dios arriba mundo GEN este mundo GEN saber CAUS AG ACUS

Lit.: 'A Dios que ha hecho saber del mundo de arriba y del mundo de aquí'.

Estamos ante cinco construcciones básicas, las primeras estudiadas y la última meramente sugerida: adjunción sin marcas $(\mathrm{S}+\mathrm{A}$ verbal), atribución directa (VCOP + A verbal), relación subordinada (SGEN + SPOSP), aposición explícita (O1 + O2 anafórica) y calificación explicativa ( $\mathrm{S}+\mathrm{ACAS}$ verbal). Es un modo sencillo de indicar que es el adjetivo derivado de un verbo el que podría valer como una oración compleja de relativo, la cual, en determinados casos, podría estar en parte separada de la estructura general bien coordinándose a un sintagma nominal (aposición oracional) o bien subordinándose al mismo mediante el caso genitivo (construcción frasal).

Para enriquecer la información dada por ST, el Anónimo de 1586 -AR por el nombre del editor Antonio Ricardo-, recurre igualmente a los relativos y, en general, a los nombres verbales.

1) Comenzando por estos, vemos que las terminaciones en - $q$ (Agente; presente), $-n a$ (futuro), -sqa (pasado) tienen la función de indicar, aparte del aspecto inherente a las 
formas verbales, que estamos ante un sustantivo (una oración completiva en ciernes) o un adjetivo complejo (una oración de relativo en ciernes): uqyana 'cosa que se puede beber', wañuna 'causa que mata', pero rikhuriq 'visible', mana rikhuriq 'invisible'.

2) Los relativos propiamente dichos están descritos con mucho más detalle y enjundia que en ST, ya que AR analiza los antecedentes pi 'quien', ima 'que' o tukuy / llapa 'todo', a partir de los cuales se constituyen. Los relativos están vistos también desde las características de tiempo y aspecto que los caracterizan. Vale la pena aducir uno de los argumentos de los autores y aportar los ejemplos con que lo sustentan:

\begin{abstract}
Toda la dificultad de los relatiuos está en estos de antecedente, porque como diximos esta lengua no tiene termino simple para significarle, y porque es proprio de los participios traer consigo estos romances, el que, la que, lo que. De aquí es que todas las oraciones de relatiuos se pueden hazer por los tres participios de presente, passado y futuro. De presente, como tú que sabes enséñame can yachac cac yachachihuay; el indio que vino de Cuzco me truxo una carta, Cozcomanta hamuc runam huc quellcacta apamuarcam; el indio que han de ahorcar me dixo que le confessasse, huarcuncapac cac runam confessahuay ñihuarcan. De preterito: al hombre que yo vi veo, ricuscay runactam ricupuni. Recibe estas palabras que hablo en tu coraçon, cay rimascay simicta soncoyquipi chasquiy. De futuro: enseñadme lo que tengo de creer, yñincayta yachachihuay; yo te enseñare lo que has de creer, yñincayquita yachachiscayqui (f. 30r).
\end{abstract}

Analicemos el primer ejemplo: Qan yachaq kaq yachachiway:

$\begin{array}{lllllllll}\text { Qan } & y a c h a- & -q & k a- & -q & y a c h a- & -c h i & -w a & -y \\ \text { tú } & \text { saber } & \text { AG } & \text { ser } & \text { AG } & \text { saber } & \text { CAUS } & 1^{\text {a OB }} & 2^{\text {a SUJ-IMP }}\end{array}$

'Tú, que eres el que sabe, enséñame'

El adjetivo verbal en forma de agente (participio de presente) vehicula la oración de relativo. Al haberse sabido encontrar la correspondencia entre los participios latinos y los del quechua, los misioneros jesuitas que hicieron el Anónimo tenían todo el camino allanado. De hecho, la semilla la había plantado ya el insigne dominico que inauguró los estudios quechuas.

Analicemos ahora un ejemplo más de pretérito y otro de futuro:

(5)

$\begin{array}{lllllllll}R i k u- & s q a & -y & \text { runa- } & -k t a & -n & r i k u- & -p u & -n i \\ \text { ver } & \text { PPAS } & 1^{\text {a}} \text { SUJ } & \text { hombre } & \text { ACUS } & \text { VAL } & \text { ver } & \text {-REGR } & 1^{\text {a SUJ }}\end{array}$

'Al hombre, en verdad, que yo había visto, he ido a ver'

(6)

Iñi- $\quad-n[q] a \quad-y k i-\quad$ ta $\quad y a c h a-c h i \quad-s q a \quad-y k i$

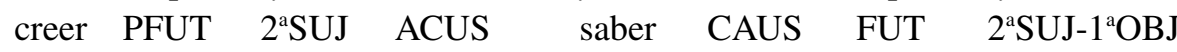

'Lo que tú tienes que creer, yo te lo enseñaré' 
En ellos se observa que el sintagma complejo en - $[k] t a$, alberga una forma de relativo que en el caso de rikusqay figura como complemento adjetivo de runa, sustantivado previamente mediante $-y$ y después adjunto como se desprende de su construcción estructural: rikuy $(\mathrm{V})>$ rikusqa $(\mathrm{A})>$ rikusqay $(\mathrm{S})$. En el último caso se trata directamente de una sustantivación sin apoyo de sustantivo alguno, que es tenida estrictamente por una transformación estructural en que iñiy $(\mathrm{V})>$ iñina $(\mathrm{A})>$ iñinayki $(\mathrm{S})$, la cual está en acusativo por hacer la función OD de yachachiy 'saber'. Son dos formas de sintaxis totalmente diferente, por tanto, pero que se presentan juntas porque ambas se asimilan al relativo y lo hacen por el paso intermedio de una adjetivación.

Además de los relativos antedichos, AR alude a los relativos siguientes:

3) Relativos oblicuos. Son aquéllos que refieren a casos diferentes del nominativo, aunque los completivos (caso - $t a$ ) aparecen incluidos en el apartado anterior. Son, pues, los de genitivo (con -yuq), los de dativo (con -paq), los de acusativo (con -ta), los de ablativo (con distintas terminaciones, según los distintos ablativos quechuas: ab-lativo -manta, lo-cativo -pi, etc.). Ejemplos:

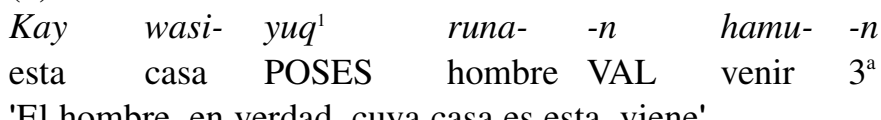

'El hombre, en verdad, cuya casa es esta, viene'

$\begin{array}{lllllllll}\text { Tiya- } & -s q a & -y k i & \text { wasi- } & -p i & -n & \text { tiya- } & -r q a & -n i^{2} \\ \text { vivir } & \text { PPAS } & 2^{\mathrm{a}} & \text { casa } & \text { LOC } & \text { VAL } & \text { vivir } & \text { PTO } & 1^{\text {a }}\end{array}$

'Viví en en la casa en la que tú viviste'

\begin{tabular}{lllllllll}
K$^{\prime}$ iri- & $-c h a$ & $-k u$ & $-s q a$ & $-y k i$ & & rumi- & - wan & $-m i$ \\
herir & CAUS & REFL & PPAS & $2^{\mathrm{a}}$ & \multicolumn{2}{c}{ piedra } & INSTR & VAL \\
$\tilde{n}$ nqa- & $-p a s$ & $k^{\prime} i r i-$ & $-c h a$ & $-k u$ & $-r q a$ & $-n i$ & & \\
yo & ADIT & herir & CAUS & REFL & PTO & $1^{\text {a }}$ & &
\end{tabular}

'En verdad que con la piedra que tú te habías herido, me herí yo también'

La única salvedad que cabe poner a la descripción de AR es la indistinción entre las formas adjetivas complejas, como las anteriores, y las sustantivas complejas, como (6), que también aparecen en la descripción y que constituyen formas sintéticas en que el adjetivo aparece definitivamente transformado a sustantivo mediante la terminación de caso.

1 Este sufijo posesivo, derivado del interiorativo $-y / k J u$ más el agentivo $-q$, se estima ahora, tanto como entonces, una forma sintética que no actúa como marcador de caso, aunque pueda traducirse por el genitivo posesivo «cuyo».

2 El autor ofrece una versión alternativa de carácter apositivo: May wasipin tiyarqanki, chaypin tiyarqani, cuyo significado literal es: 'En la casa en donde viviste, [en] allí, en verdad, viví yo'. Obsérvese la presencia de los dos locativos correlativos en - $p i$. 
4) Relativos de consecuente. En base al tratamiento del tema por parte de ST, AR nos recuerda el modo de explicar los relativos con antecedente kay, chay y kikin. El ejemplo Iesu Christo paypa churinmampas, caymi Espiritu Sanctomanta runa tucurqan 'Y [creo] en Jesu Christo, [que es] su vnico hijo, el qual fue concebido por el Espíritu Sancto' muestra las posibilidades de la aposición, que sólo formaría supuestos relativos partiendo de dos premisas controvertidas: 1) la confusión de la anáfora kay 'este' con el relativo estricto y 2) la creencia de que la traducción a otra lengua, que exige en este caso construcción relativa, hace sin más que se igualen. Una tercera inexactitud, propiciada también por la relativa indistinción adjetivo / sustantivo en quechua, es que formas como la siguiente, con adjetivo transformado, se consideren relativas:

(10)

Huq- $\quad-n i \quad-y k i$

uno ESTR $2^{\mathrm{a}}$

'Uno de vosotros'

La razón de llamarlas oraciones de consecuente, en este caso, es la no presencia de un antecedente explícito en la oración.

5) En último lugar, AR trata los relativos de accidente, que se suplen por las partículas chhika 'pedazo, porción', hina 'así'. Tal es el caso de:

$\begin{array}{lllllll}\tilde{N} u q a- & -n & \text { rit'i } & \text { hina } & \text { yuraq } & k a- & -n i \\ \text { yo } & \text { VAL } & \text { nieve } & \text { así } & \text { blanco } & \text { ser } & 1^{\text {a }}\end{array}$

'Yo, en verdad, soy blanco como la nieve' (lit.: 'yo [que soy] como la nieve, soy blanco')

$\begin{array}{llllllll}\text { May } & \text { chhika- } & -n & -m i & \tilde{n} a w i- & -y k i & & \\ \text { cual } & \text { porción } & \text { VAL } & \text { VAL } & \text { ojos } & 2^{\mathrm{a}} & & \\ \text { chay } & \text { chhika- } & -n & -t a q & -m i & \text { rinri- } & -y k i & - \text { pas } \\ \text { eso } & \text { porción } & \text { VAL } & \text { CONTR } & \text { VAL } & \text { orejas } & 2^{\mathrm{a}} & \text { ADIT } \\ \text { 'Tantos cuantos son tus ojos son tus orejas'. } & & & \end{array}$

La extensión de aquello que se tenía por relativo en el Renacimiento y lo que se considera más restrictivamente como tal ahora no coinciden. Aquí se siguen de cerca, también extensivamente, las gramáticas latinas en sus aspectos más formales. Alvares (1572) en su tratamiento del pronombre relativo latino (ff. $9 \mathrm{v}-10$ ) señala cómo los distintos gramáticos latinos fueron incorporando diversos pronombres a este conjunto y cómo algunos autores los consideraban más bien entre los nombres. Autores como Probo, Diomedes, Donato, Servio abundan en su valor pronominal, aunque Varrón se sale de la doctrina común para incorporarlos al conjunto de los comodines (prouocabulum 'provocablo') de carácter finito (de los nombres propios) o infinito (de los comunes). Entre estos pronombres no sólo están qui, quć, quod, sino también quantus, tantus, qualis, talis (de Probo), cuius (de Diomedes), tot...quot (de Donato), etc., de modo que las glosas latinas de los ejemplos quechuas antedichos los recogen sin excepción. Por ejemplo (12), que no se 
glosa en castellano sino en latín, es Tot sunt oculi tui qout aures. Otras formas pronominales compuestas como quisque, demostrativas como iste, indefinidas como alius 'otro entre varios', alter 'otro entre dos' o reliquus 'los demás' (en quechua wakin) se incorporan también al conjunto descriptivo, a la hora de entender y clasificar los relativos en quechua:

$\begin{array}{llll}\text { Waki- } & -n & -n i & - \text { nchiq } \\ \text { los demás } & \text { VAL } & \text { ESTR } & 1^{\text {aPL-INCL }} \\ \text { 'Los demás de nosotros' = 'algunos de nosotros' }\end{array}$

Si bien Nebrija, en su Gramática (1492, cap. II), alude a las diferentes clases de relativos: de sustancia por referirse a un sustantivo (quien) y de accidente por hacerlo a un adjetivo (tal, tanto), es la tradición latina en todo su desarrollo la que sirve por igual no sólo a la gramática castellana, sino a la del quechua y a la de la mayoría de las lenguas indígenas de América. Todo el grueso de esta doctrina se ha venido llamando Gramática Tradicional, que es la que como paradigma aplican con rigor y exactitud los gramáticos de las lenguas europeas y la que se extiende prácticamente de este modo hasta la segunda mitad del siglo XX. Respecto a las lenguas indígenas, tras la luminaria inicial de ST, es la escuela de Lima (AR) la que consigue configurar este fenómeno del relativo tanto en quechua como en aimara. Tan es así, que la más brillante de todas las escuelas de lenguas indígenas de América del Sur, la Escuela de Juli, la que dio las mejores gramáticas del quechua y el aimara y también del mapuche y del guaraní, a partir de 1603, no logró superar a aquélla en diversificación por lo que al relativo se refiere, aunque termina de configurarlo. Diego González Holguín -GH a partir de ahora- dado lo monumental de su obra, hace aflorar por doquier el tema de los relativos y trata de forma exhaustiva todos y cada uno de los compuestos latinos y de su correspondencia con el quechua, profundizando teóricamente en la descripción del fenómeno, y separando cualitativamente los diversos fenómenos. A nivel descriptivo, GH distingue tres tipos de relación entre el adjetivo y el sustantivo:

1) El adjetivo se une íntimamente al sustantivo anteponiéndosele y forma un sintagma complejo: sinchi runa 'hombre fuerte'.

2) El sustantivo por vía de «construcción o régimen» se une a otro sustantivo que lo rige. La rección se produce en genitivo: huasi-p punku-n 'la puerta de la casa' (lit.: casade puerta-su), la cuál admite alguna partícula intermedia como en hanac pachappas cay pachappas ruraquen 'hazedor del cielo y la tierra', en que se introduce el aditivo -pas 'también' tras el genitivo $-p$ (hoy $-q$ ).

3) Un sustantivo antepuesto, generalmente de materia, se une directamente a otro pospuesto y entonces ejerce de adjetivo directamente, trasladando el problema al apartado 1): cullqui aquilla 'vaso de plata' (lit. plata vaso).

Una nueva relación, establecida como en 2) entre dos sustantivos, es aquélla en que los dos núcleos están en «aposición», sin que se refieran a dos cosas diferentes del mundo externo («cuando uno se pone para declarar a otro») como en Iesu Christo Dios en «Diospa churin, Iesu Christo Dios 'Jesucristo Dios, que es hijo de Dios'» (f. 4r). En este caso el sustantivo no se adjetiva -señala GH- y ello -añadimos nosotros- pese a que la 
traducción que ofrece es una oración de relativo: los dos fenómenos, construcción genuina y traducción, habían quedado separados. ${ }^{3}$

La anterior no es meramente una impresión mía, sino que acto seguido (lib. I, cap. III: de la declinación de los participios adjetivados) GH presenta las formas de declinación con participios como en Hanac pacha cay pacha rurac Dios 'Dios que hizo cielos y tierra', cuyo análisis es como sigue:

$\begin{array}{lllllll}\text { Hanaq } & \text { pacha } & \text { cay } & \text { pacha } & \text { rura- } & -q & \text { Diyus, } \\ \text { arriba } & \text { mundo } & \text { este } & \text { mundo } & \text { hacer AG } & \text { Dios }\end{array}$

en que - $q$ es el agente que construye adjetivos activos en quechua (modelo 1), o bien construcciones de régimen como Hanac pachap ruraquen (o ruracnin) ${ }^{4}$ 'El hazedor de los cielos' (modelo 2):

$\begin{array}{llllll}\text { Hanaq } & \text { pacha } & -q & \text { rura- } & -q i & -n \\ \text { arriba } & \text { mundo } & \text { GEN } & \text { hacer } & \text { AG } & 3^{\text {a }}\end{array}$

'De los cielos su Hacedor'

En efecto, cuando GH aborda el tema de los sustantivos genitivizados, que suelen traducirse por frases relativas a español, deja de lado el problema de estos, dado que no se rebasa el campo de la categorización sustantiva, como en: runap (hoy runaq: runa 'hombre', $-q$ 'GEN') 'lo que es del hombre', existiendo en quechua sobredeclinación acumulada como en runapapninhuan 'Las cosas que son del hombre', cuyo análisis es:

$\begin{array}{lllll}\text { Runa- } & -p a q & -n i & -n & - \text { wan, } \\ \text { hombre } & \text { BENEF } & \text { ESTR } & 3^{\text {a }} & \text { INSTR }\end{array}$

en que el benefactivo queda subsumido en la sustantivación mediante la tercera persona - $n$, sustantivadora, sustantivo complejo declinado en instrumental -wan, cuyo equivalente exacto en español es 'con lo que es para el hombre'. De hecho, GH niega incluso que los sustantivos posesivizados, acabados en -yuq, sean adjetivos: qullqiyuq 'el que tiene plata' (qullqi 'plata', -yuq 'POSES'), criticando con ello la decisión de incluirlos entre los relativos por parte de los gramáticos de la Escuela de Lima (ej. 7).

En GH hay que ir directamente a los pronombres relativos (lib. I, cap. VI. 4) para descubrir el meollo de la construcción sintáctica correspondiente. Distingue pi 'quien' para

\footnotetext{
3 En Pedro huaccha, que podría equivaler por la traducción (Pedro 'el pobre') a la secuencia S $+\mathrm{A}, \mathrm{GH}$ habla también, directamente, de aposición. Y lo mismo sucede en el caso en que tenemos Pron. $+\mathrm{S}$, en que se invierten los términos: cam yayay 'tú que eres mi padre' (cam 'tú', yaya 'padre' - $y$ ' $1^{\mathrm{a}}$ ').

4 Rura-q-ni-n, con el estribo eufónico - $n i$, responde al mismo esquema.
} 
personas, yma 'que' para cosas y maycan 'cual, cualquier' común a todos, sin añadir novedad práctica alguna al tratamiento quechua. Especulativamente, en cambio, abre la posibilidad a un tratamiento más amplio al indicar lo «difficultoso [que] es averiguar en esta lengua quales sean adjetivos y substantivos, porque un mismo nombre lo suele ser todo» (f. 13r). De ahí que al tratar el tema de la adjetivación de los pronombres (ñoca cinchi 'yo fuerte, yo que soy fuerte') como el de la composición del pronombre con el participio (munascay 'lo amado de mí', munascayqui 'lo amado de ti', f. 18r) da de hecho pistas para tratar teóricamente en quechua el fenómeno de la relativización.

Pero es en el capítulo concreto que versa sobre «las oraciones relativas» (lib. IIII, cap. IX, X y principalmente XI; ff. 128-132) donde nos encontramos con todas las aportaciones de interés y las consecuentes acotaciones de campo. Como era de suponer, busca ante todo las correspondencias morfológicas entre el latín, el español y el quechua del tipo de las que van a continuación:

\begin{tabular}{|c|c|c|}
\hline QUIS & pim / pillam & quien \\
\hline QUISNAM & pich / pillach & quienquiera \\
\hline QUIDAM & huc / hucca & uno / el uno \\
\hline QUISQUE & pipas / pillapas & cualquiera \\
\hline UNUSQUISQUE & hucpipas / hucpillapas & uno cualquiera \\
\hline $\begin{array}{l}\text { QUILIBET } \\
{[\ldots]}\end{array}$ & pi munascapas & qual / quienquiera que \\
\hline QUID & yma / ymalla & que cosa \\
\hline QUIDQUID & ymapas / ymallapas & qualquiera cosa \\
\hline QUODCUMQUE & ymapas / haycapas & qualquiera cosa que \\
\hline $\begin{array}{l}\text { QUALECUMQUE } \\
{[\ldots]}\end{array}$ & maycan imapas & qualquier[a] cosa de qualquier modo o manera \\
\hline QUALIS & maycan / maycallam & qual persona o qual cosa \\
\hline QUALISNAM & maycancha & no se qual \\
\hline $\begin{array}{l}\text { QUALISQUISQUE } \\
{[\ldots]}\end{array}$ & maycanpas & qualquiera \\
\hline
\end{tabular}

Posteriormente señala los tipos de oraciones de relativo encontradas, «las quales se hazen por los relatiuos o por verbo; ya con nombres relatiuos ya sin ellos» (f. 131r), que son:

1) «Todos los participios de su naturaleza y propria significacion son relatiuos» (ibíd.)

2) «Por los verbos» (ibíd.), que es manera más «galana».

3) «Con los nombres relatiuos (pi, yma, maycan...)» (ibíd.). Esta manera es común a las dos primeras con lo que «no ay más de dos maneras de relaciones, o por participios, o por verbos, porque los nombres relatiuos no hazen oraciones por si [...] sino allegandose a vno de estos dos» (ibíd.).

Así tenemos del primer modo:

Diospa gracianta chazquicunanpac camaricuc runaca, chayca usachicunca 'el que se dispusiere para recibir la gracia de dios la alcançará', pudiéndose también formar con pi: Diospa gracianpac pi camaricuc runaca 'el hombre que se dispone a la gracia'. Veamos el análisis parcial: 
(17)

$\begin{array}{llllllllll}\text { Diyus } & -p a & \text { gracia } & -n & -t a & \text { chaski- } & -k u- & n a & -n & -p a q \\ \text { Dios } & \text { GEN } & \text { gracia } & 3^{\text {a }} & \text { ACUS } & \text { recibir } & \text { REFL } & \text { FUT } & 3^{\text {a }} & \text { BENEF } \\ \text { kama- } & -r i & -k u- & -q & \text { runa- } & -q a & & & & \\ \text { mandar } & \text { INC } & \text { REFL } & \text { AG } & \text { hombre } & \text { TÓP } & & & & \end{array}$

'En cuanto al hombre que se dispone a recibir la gracia de Dios'

(18)

$\begin{array}{lllccc}\text { Diyus } & -p a & \text { gracia } & -n & -p a q & p i \\ \text { Dios } & \text { GEN } & \text { gracia } & 3^{\text {a }} & \text { BENEF } & \text { quien } \\ \text { kama- } & -r i & -k u- & -q & \text { runa- } & -q a \\ \text { mandar } & \text { INC } & \text { REFL } & \text { AG } & \text { hombre } & \text { TÓP }\end{array}$

'En cuanto al hombre, el cual se dispone para la gracia de Dios'

Con ello hemos entrado en la división, ya clásica en quechua, entre el adjetivo verbal que forma relativos y la verdadera aposición marcada mediante la adjunción de $p i$ (como en latín qui se prćparat o is qui se prćparat).

Eliminado el sustantivo que sirve de apoyo al adjetivo, tenemos los relativos con verbo como Diospa gracianpa pipas camaricun, cuyo análisis es el que sigue:

(19)

Diyus -pa gracia $-n$-paq pi $\quad$-pas kama- $-r i-{ }_{-}-k u \quad-n$, Dios GEN gracia $3^{\text {a }}$ ACUS quien INDEF mandar INC REFL $3^{\text {a }}$

'Quienquiera [que] se prepara para la gracia de Dios'

O las construcciones con el tópico -qa, antecedido del demostrativo chay 'este', etc., como en Diospa gracianpac camaricun chay runaca 'en cuanto a este hombre [que] se prepara para la gracia de Dios' o bien Diospa gracianpac camaricun chayca 'en cuanto a este [que] se prepara para la gracia de Dios' en que la elipsis no obsta para la consideración de una oración relativa intrínseca. La dificultad estriba en que se incumple la norma del adjetivo, por un lado, y en que la construcción es más bien, la de una subordinada bimembre, no incrustada, de significación plural, a tenor del contexto. Así las dos formas de abajo, se pueden traducir tanto por una oración de relativo como por una subordinada, temporal, causal o condicional:

(20) Diyuspa gracianpaq kamarikun chay runaqa, usachikuntaqmi

'El hombre que se dispone // Cuando el hombre se dispone / Porque el hombre se dispone / Si el hombre se dispone a la gracia de dios, la alcanzará'.

(21) Diyuspa gracianpaq kamarikun chayqa, usachikuntaqmi

'El que se dispone // Cuando se dispone / Porque se dispone / Si [alguien] se dispone a la gracia de Dios, la alcanzará'.

Es precisamente la partícula tópica - qa la que hace de frontera y la que incide en la posterior consideración de la bipolaridad oracional encontrada. Como GH reconoce, otras partículas como -si, -mi, etc. también servirían al efecto. El avance o acotación del problema, como hemos visto con cierto pormenor, ha proseguido con $\mathrm{GH}$, pero el conocimiento global de las estructuras de relativo requería aún futuros esclarecimientos. 


\section{El desarrollo actual y los antecedentes misioneros}

En los últimos desarrollos de la lingüística, el relativo ha tenido un fuerte protagonismo. Sin entrar en detalles estrictamente monográficos, el estructuralismo ha insistido en la distinción entre la oración de relativo restrictiva y la no restrictiva, o lo que es lo mismo, en la acotación de la referencia del SN correspondiente: La mujer que es joven viste más desenfadadamente, pero La mujer, que era joven, salió corriendo del lugar, aspecto que no había sido tenido en cuenta en los estudios quechuas de los misioneros. Sólo desde una perspectiva pragmática, y por tanto del uso, cabe aproximarse a este asunto, por lo que no extraña que los misioneros, que remitían constantemente a él para no ser prolijos, dejaran de lado la distinción. Por otro lado, dado que el artículo no existía en quechua y que ésta es la categoría básica de reconocimiento de la referencia, las dificultades se acrecentaban. No obstante, el constante recurso a las «partículas de ornato»o «de elegancia» por parte de los gramáticos quechuas de los siglos XVI al XVIII, como el tópico - $q a$, el evidencial - $m i$, el conjetural -chá, el reportativo -si, pudo haber propiciado este análisis, aunque fue la asignatura pendiente de los misioneros, que no observaron que bajo los efectos retóricos de estos u otros enclíticos, se escondían fenómenos pragmáticos de muy profundo calado. No obstante, hay aproximaciones cuidadas a los mismos que nos proporciona tanto GH como Diego de Torres Rubio (1619) -desde ahora TR. El primero, pese a las dificultades señaladas, recurre con insistencia a $-m i$ y a $-q a$, a la hora de explicar de los relativos:

Digo que vna misma voz puede significar dos cosas en diferente composicion, y hazerse de interrogatiua relatiua porque aqui no pregunta sino haze officio de relatiuo, y la composicion y señal de que (pi) no es interrogatiuo, sino que se muda en relatiuo es la (ca, y chayca) que ha de hauer en cada oracion relatiua, y quando la ay ya pi, o yma se haze relatiuo. Yten los interrogatiuos no se hallan $\sin (\mathrm{m})$ o (mi) que es su señal, y aqui quando (pi) es relatiuo no puede tener (m) ni mi (131v).

TR se centra sobre todo en - qa y el compuesto chayqa, cuando comenta que los relativos se hacen por los tres participios de presente, pasado y futuro (-sqa, $-q$ y $-n a)$ o por los tiempos verbales correspondientes, comentando respectivamente:

añadiendoles esta particula ca, que es la que hace la relacion, á la qual lia [sic] de responder immediatamente este pronombre chaycca (fol. 32v) / añadiendo al verbo la dicha particula cca, a la qual le ha de corresponder immediatamente el pronombre [c]haycca (f. 33 r). ${ }^{5}$

Pero lo hace sin penetrar en el ámbito de la referencia de los sintagmas involucrados. Pese a todo, esta no sería la solución ya que el uso de - qa no garantiza la genericidad o especificidad del relativo. Así «sermon vyariscayquitaca villahuay, quentame el sermon que has oydo» que exige -qa según GH (f. 132r), forma una oración restrictiva, mientras que los demás ejemplos que aporta se mantienen, con la misma partícula, en la genericidad no restrictiva y significan «el que, la que». De igual modo, cuando la partícula acompaña

5 Así se dirá, en efecto -sigo la ortografía del original-: runa Diospa acllascancca chaecca hanacpachaman rinccan I Diospa acllascan runacca hanacpachaman rinccan 'el hombre escojido de Dios irá al Cielo' (con participios) o ttanta micuchcanicca, chaycca yuracmi 'el pan que estoy comiendo es blanco' (con verbos). 
al demostrativo chay «ese» como en chayqa, chaymi, chaysi, chayta, etc. se hace «conforme al sentido» (f. 132v), sin que éste se precise con exactitud por parte de GH en el desarrollo temático. He aquí, pues, que queda una asignatura de importancia pendiente en la descripción del relativo por parte de los gramáticos misioneros del quechua. ${ }^{6}$

Por otro lado, en el ámbito de la gramática relacional, el descubrimiento de Keenan (1972, Keenan \& Comrie 1977) de la escala de accesibilidad al relativo cuyo orden universal es: sujeto $>$ objeto directo $>$ objeto indirecto $>$ objeto preposicional $>$ objeto posesivo > objeto comparativo, vino a revolucionar desde entonces los estudios sobre los relativos quechuas (Weber 1976, 1983). Casi nada de esto era previsible en la teoría lingüística del Renacimiento. La discusión se centra hoy principalmente en la delimitación del terminativo -sqa y el prospectivo -na, que difieren del activo - $q$ : éste se mantiene más bien al margen del tiempo, manifestando en su caso un aspecto durativo o puntual a tenor de la semántica del verbo. Por otro lado, se debate la existencia o no de relativos con participio de presente agente en $-q$ fuera de la posición del sujeto (Costa 1972, Weber 1976, Lefebvre \& Muysken 1982), pero los autores misioneros que hacen una distinción estricta entre el infinitivo o nombre verbal $-y$ y los sufijos adjetivales, no tienen en principio demasiada conciencia de estos fenómenos. Pero es algo más que la intuición lo que lleva a un autor algo más tardío, IRMO (1648) -que responde al nombre del cura cuzqueño Iván Roxo Mexía y Ocón- a proponer soluciones sorprendentes mediante el cotejo sistemático de tres lenguas: latín, español y quechua. Resumiendo la postura de hoy en día, la estructura de los sufijos nominalizadores, ${ }^{7}$ que proporcionan adjetivos y forman relativos en quechua, sería la siguiente:

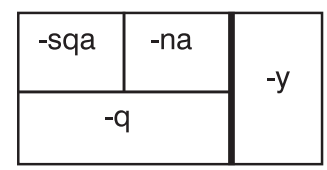

lo que contrasta con Calvo Pérez (1993, 295):

\begin{tabular}{|c|c|c|c|}
\hline & PRETÉRITO & PRESENTE & FUTURO \\
\hline NOMBRE/VERBO & \multicolumn{3}{|c|}{-y (atemporal positivo) } \\
\hline VERBO $\rightarrow$ NOMBRE & -sqa & $-q$ & -na \\
\hline NOMBRE-VERBO & --- & oa & \\
\hline
\end{tabular}

${ }^{6}$ Es precisamente una de las partículas de ornato, el limitativo -lla, la que influye directamente en la acotación citada. Así: Karu tiyaq yachaqkunalla qhipayukunku '[sólo] los alumnos que vivían lejos se retrasaron' / Karu tiyaq yachaqkuna qhipayukunku 'los alumnos, que vivían lejos, se retrasaron'. Los lingüistas actuales, puesto que se dedican casi exclusivamente a las relativas especificativas (primer tipo), dejan de lado las explicativas (segundo tipo). GH, que alude a las funciones expresivas de -lla, señala que significa 'solamente' "como runalla solo el hombre» (f. 106v), pero no aplica este conocimiento a la discriminación referencial del relativo, pues en el capítulo correspondiente (IX del libro IIII) apenas si comenta que «en todos los compuestos de (pi) y (yma) entra (lla) y las aumenta casi sin significación» (f. 129r).

7 Autores como Costa (1972) o Snow (1973) asumen que la relativización en quechua implica nominalización. Calvo (1993), también, excepto en los casos en que se mantiene la forma aposicional en que hay un predomino del nombre sustantivo sobre el nombre adjetivo (tan próximos en quechua). Esta parece ser también la postura de los misioneros, sobre todo la de GH, que habla frecuentemente de aposición (f. 6r, etc.). 
en que prefería la proyección extralingüística del tiempo en tres dimensiones aspectuales internas y se cotejaba con la sintáctica: la presencia de un solo sujeto para los casos de -spa $\mathrm{y}$, eventualmente, de -qti y la expandida del obviativo para casos de no coincidencia de sujeto (también con -qti). Los autores generativistas prefieren generalmente separar - $q$ de las demás formas nominalizadoras, pero reconocen al tiempo que hay excepciones como en Warma Husichawan rimaqwan / Husichawan rimaq warmawan pukllarani 'jugué con el muchacho con quien habla / que habla con Pepito', donde se desarrolla un sujeto interstancial oblicuo o en [payta] asiqta uyarini 'le oí [a él] riéndose', en que hay implicado un objeto directo calificado aposicionalmente en el ámbito de un verbo de percepción; o bien en algunos ejemplos de mi corpus (Calvo Pérez en pr.):

(22) Uruwachiqta wakata apashani 'estoy llevando <a que la coja el toro > a la vaca que está en celo'

(23) Ñuqaqa payta paqarin tiyaqta kachisaq 'yo a ése lo dejo sentado <en el baile> mañana'

(24) Suwashaqta tarirunku, hinaspa paywan tukupunku 'han cogido al que estaba robando y por eso lo han linchado'

(25) Wañuchiyta riqsichinku warmi rutuna[ta] hap'ishaqta hina 'simbolizan a la muerte como una mujer con una guadaña en la mano'

(26) Karrupi sini qhawakuqman risaq 'iré al autocine' (lit. 'hacia el sitio en que se ve el cine en carro')

(27) Mana kaypi kaqkunata manan ninichu 'no me gusta mentar [= no miento] a los ausentes [= a los que no están aquí]'

(28) Manan chaskinichu huqmanta rimakuqta 'no recibo <en casa > a los que difaman a otros'

(29) Arkiruqa llapan piluta chayaqta hark'aran 'el meta paró todas las pelotas que le llegaron'

(30) Warmakuna armakuq rirqanku llaqta sispa puriq mayupi 'los muchachos iban a bañarse al río, que pasa cerca de la ciudad'. ${ }^{8}$

Los autores clásicos, los del Renacimiento naturalmente, no sólo distinguieron la diferencia de formas para la coincidencia / discrepancia de sujetos (lo que AR llama «supuestos», f. 11v), sino que en ocasiones supieron aproximarse al problema diferencial de $-q$ (con ortografía $<c c>$ ) frente a los demás adjetivos verbales. IRMO, que dedica un espacio importante al tema del relativo (ff. 41-48), percibe, creo que con meridiana claridad, este problema:

Todas las vezes que el relatiuo qui es nominatiuo, como el que, la que, lo que, siempre se hace la oracion por el participio de presente, aunque sea el romance de preterito, futuro o circunloquio del qualquiera modo indicatiuo o subjuntiuo (f. 44 r) ${ }^{9} /$ Cuando el relatiuo

8 Ello no es óbice para que aceptemos que es la calificación del sujeto la que acapara la mayoría de los ejemplos existentes, sobre todo si se dan en cascada, como este ejemplo de Soto que cita Wölck (45): «chakrapi wiñaq sara(ta) mikuq wallpa(ta) suwaq warmatam rikurqa 'vio al muchacho que se robó la gallina que había comido el maíz que crece en la chacra'» dicho con «desparramamiento» a la izquierda.

9 Pese a la mala conservación del original (f. 44v), reconstruimos uno de los ejemplos tomado del Evangelio de Lucas 2, 18: «Omnes qui audierunt, mirati sunt. 1 Todos 2 los que oyeron 3 se admiraron / 1 Hinantin 2 uyaricccuna 3 utirayarccan». 
qui es acusatiuo, o caso de verbo, se haze la oracion en la lengua por el participio en scca del verbo a quien se junta de qualquier tiempo y modo que sea el romance $(45 \mathrm{r})^{10}$ / Se aduierta que los tiempos de futuro, donde el relatiuo qui es acusatiuo, se puede hazer con la misma propiedad por el participio de futuro en $n c c a$ [hoy -na] f 46r). ${ }^{11}$

Abundando en lo anterior, IRMO recapitula después en su «suplemento al participio» (f. 46r-v) y hace de nuevo hincapié en lo dicho. ${ }^{12}$ Por diversas razones, el análisis en profundidad de esta obra, cuyo objetivo básico es la traducción de la Biblia al quechua, nos depara más de una sorpresa sobre las categorías de esta lengua andina, siempre con el criterio, intuido por mí, de que tales observaciones no pueden haberse hecho si no es aplicando rigurosamente el método experimental (estructural) y no sólo haciendo uso del mecanismo distribucional que IRMO exhibe a lo largo de toda la obra.

La Gramática Generativa, globalmente considerada, explotó enseguida una de las primeras transformaciones que llevarían de la estructura profunda oracional (La mujer es joven) a la adjunción adjetiva (La mujer joven / La joven mujer), pasando por el estadio intermedio de la relativización (La mujer que es joven). Las cosas fueron así a partir de 1960 como consecuencia de las regularidades transformacionales observadas en este proceso. Nada de esto tenía tampoco antecedentes explícitos en el tratamiento del relativo quechua, si bien los misioneros, siguiendo la tradición latina, hacen la oportuna distinción entre las oraciones relativas y las funciones diferenciales de sustantivo y adjetivo, asociándolas debidamente entre sí. De hecho la Gramática Tradicional y luego la Estructural o Funcional se debaten entre la descripción de los relativos / interrogativos en sí mismos y el análisis de la función que han de desempeñar después en la oración (Gili Gaya, 1973, Alarcos 1994; para el quechua Wölck, 1987³).

Por otra parte, el desarrollo de la deixis y el paralelismo establecido entre ella y otras muchas categorías gramaticales por la Pragmática Topológico-Natural (Calvo Pérez,

${ }^{10}$ Por ejemplo, del Evangelio de Lucas 11, 27 (f. 45v): «Beatus venter, qui te portavit, et ubera, quae suxisti. 1 Bienaventurado 2 el vientre 3 que te tuvo en si 4 y 5 los pechos 6 que mamaste / 3 apariccñijqui 2 huicça 5 ñuñu 6 ñuñusccayquicuna 4 pas 1 añay munayllam».

${ }^{11} \mathrm{Y}$ añade ejemplos evidentes de nuevo como éste del Evangelio de Mateo 20, 22 (f. 46r): «Potestis bibere calicem, quem ego bibiturus sum? 1 Podéis 2 beber 3 el cáliz 4 que yo tengo que beber? / 3 calis 4 uppianccayta (o 4 uppianayta) 2 uppiayta 1 atipanquichicchu».

12 Habríamos esperado que IRMO asociara el desarrollo del participio en - $q$ al clasema de los sintagmas implicados en la concordancia con él, como lo hace al distinguir los pronombres relativos pi y may (para personas) de ima (para cosas). Pudo ser una omisión o bien la duda que esta distribución genera al respecto. Para Costa (1972: 71) «in a relative clause [with $-q$ ] the noun referred to need not be animate" como en puriq mayupi "en el río que discurre»; para Weber (1983: 31-33), en cambio, en estos casos la estructura no es sino en apariencia la de una frase frase nominal relativizada. Parece entonces que $-q$ es siempre un sufijo Agentivo, pero la experiencia demuestra que no es así (al margen de su comportamiento como Genitivo mamaykiq wasin 'la casa de tu madre'). Para mí ( $N D$ en prep.), - $q$ abarca también al Experimentador y al Locativo, y al Instrumental (como -nte en español: amante / saliente / cortante), por el que se traduce también, y, por supuesto, al más activo, al Agentivo o Instrumental (como -dor en español: matador / picadora). Hay casos, no obstante, en que - $q$ es una forma anquilosada del pasado o invoca un comportamiento al margen de la voluntad: sumaq 'hermoso', llasa-q 'pesado', illa-q 'brillante'.

${ }^{13}$ Este último dice para la lengua andina: «Aunque el quechua no ha tenido un pronombre relativo, que sería necesario para la conexión de una verdadera frase verbal relativa a una constituyente de la oración principal, tenía desde luego la posibilidad de emplear su pronombre interrogativo pi 'quien' para la introducción relativa demostrativa, igual que el castellano, pero con algún sufijo dubitativo, como en el ejemplo tomado de Middendorf: Pichá mana 'kepayta munanchu chaika ripuchun 'Quien (= pichá) no quiere quedarse (este = chaika) puede regresar'» (Wölck, 1987: 90). 
1993 y 1994) viene a correlacionar los pronombres quechuas pi 'quien', ima 'que' y may 'cuál' no sólo con los rasgos de persona o cosa, sino con el variable alejamiento cognitivo del eje de coordenadas deícticas (López García, 1994). De ahí la eficaz presencia del deíctico neutralizador de distancias, chay 'ese' en las estructuras oracionales quechuas. Es decir, que si bien los misioneros no determinaron los relativos en todo su rigor, sí supieron tener la intuición debida para no alejarse del camino y abrir posibilidades a los estudiosos futuros.

Me serviré de esta última afirmación para hacer constar que muchas de las clasificaciones que hacen hoy los lingüistas del quechua estaban ya de hecho -latentes o patentes- en los estudios misioneros. También las dudas que ahora mismo se suscitan. Por ejemplo, el generativista Weber (1976) clasifica los distintos tipos de relativización del siguiente modo:

1. Relativización por yuxtaposición. El verbo subordinado relativo lleva los mismos sufijos de tiempo y persona que cualquier verbo no subordinado (runa maqa-šku-šu-nki čay čiki-nki 'odias al hombre que te ha pegado', para maqa- 'pegar' y čiki- 'odiar'; lit.: 'hombre te.ha.pegado este odias').

2. Relativización con pronombre interrogativo (mana rika-ška-:-ču pi maqa-šku-šu-nki 'no he visto al que te pegó'; lit.: 'no he.visto quien te.pegó').

3. Relativización básica (rika-ška-: qu-šu-na-ykita 'he visto lo que te va a dar'; lit.: 'he.visto la.cosa.que.te.va.a.dar', con el sufijo nominal -yki en vez del verbal -nki). A este grupo se añadiría la secuencia $\mathrm{A}+\mathrm{S}$, cuando $\mathrm{A}$ es un adjetivo verbal (čayraq čayamu-sqa runakunata wakyamuy 'llama a la gente que acaba de llegar'; lit.: 'al.instante llegados a.los.hombres ve.a.llamar').

4. Relativización con chay ${ }^{14}$ (may-pa-mi čiri-n ka-š(q)a čay-lla-pa muru-paku-n 'solamente siembran donde haya sido fertilizado'; lit.: 'en.donde fertilizado haya.sido en.esto.nomás siembran').

Permítaseme un comentario anecdótico que serviría para evaluar globalmente la aportación misionera: la lectura detallada de GH daría también pie para adoptar de inmediato la relativización apuntada en 4. Las demás también aparecen discutidas, sin excepción, en la bibliografía misionera tal y como vimos en la primera parte de este ensayo.

Por su parte, Lefebvre \& Muysken (1982) distinguen dos tipos mayores de frases relativas en quechua:

1. Nominalizadas. El verbo se hace adjetivo verbal para poder estar subordinado o para funcionar como adjetivo junto a un nombre (riku-sqa-y warma-qa hamu-nqa 'la chica que vi vendrá'; lit.: 'vista.por.mí en.cuanto.a.la.chica vendrá'). La frase nominalizada, entre otras variaciones, puede sufrir incorporación del relativo al conjunto nominal (warma rikus-sqa-y-ta-qa hamu-nqa 'la chica que vi vendrá'; lit.: 'chica en.cuanto.a.la.vista.por.mí vendrá').

2. No nominalizadas.

2.1. El verbo como tal se halla en una cláusula subordinada, la cual se reconoce por el pronombre relativo ( $r i k u$-ša-ni warmi-ta, pi-n hamu-n 'estoy viendo a la mujer, quien ha venido'), por algún demostrativo apuesto (warmi hamu- ša-n čay-ta, čay-ta-puni riku$n i$ 'he visto a la mujer que viene'; lit.: 'la.mujer está.viniendo esto, esto.verdaderamente

${ }^{14}$ Presente en un apéndice, añadido posteriormente a la redacción del ensayo. 
he.visto'), por el tópico (warmi-ta riku-ni čay-qa, pay-mi rima-wa-nqa 'he visto a la mujer, ella me hablará' = 'la mujer que he visto me hablará'; lit.: 'la.mujer he.visto en.cuanto.a.esto ella.en.verdad me.hablará'), por pronominalización (warmi riku-qti-y-mi, paymi rima-wa-nqa 'en verdad que la mujer que vi, ella me habló') o por coordinación (čay allqu-ta-qa, Mariyača qhawa- ša-n, paqarin-qa Huwanča čay-ta ranti-nqa 'mañana, Juan comprará el perro que María está mirando'; lit.: 'a.ese perro.en.cuanto.a María está.viendo, en.cuanto.a.mañana Juan a.ese comprará').

Tampoco en este caso cabe insistir mucho más: las sucintas aportaciones que he dado más arriba, aseguran que esta clasificación es la misma a que reduce GH sus cláusulas relativas. Recuérdese, si no, su afirmación sin paliativos: «...y assi no ay mas de dos maneras de relaciones, o por participios o por verbos, porque los nombres relatiuos no hazen oraciones por si ni por otro modo, sino allegandose a vno de estos dos». (f. 131r).

Un tercer y último apartado, por lo que toca a este ensayo, lo constituiría el conjunto de estrategias que tiene el quechua para hacer relativos, que son muchas por carecer esta lengua de pronombre específico. Ello ha traído como consecuencia que se discuta si hay una única forma universal de encriptar el relativo como cree Keenan (1972), debiéndose la realidad plural encontrada a un problema de núcleo y periferia o de cumplimento de alguno o todos los parámetros de la relativización (aspecto discutido por Weber 1976 y 1983) o bien si existen varias irreductibles a una sola. Los misioneros, en el fondo, también recogen este problema: por un lado tratan el relativo como un todo, con orientación desde de los relativos del latín y sus correspondencias con el quechua, pero al mismo tiempo son capaces de discernir como GH las dos formas que describe Lefebvre \& Muysken (1982). Damos la razón a estos últimos, frente a Keenan y Weber, en el sentido de que una gramática profunda muy reducida es un aparato que sintéticamente puede parecer un logro importante, pero que en realidad distorsiona la pluralidad congénita de las estructuras del lenguaje. Y en eso el quechua ha demostrado ser una lengua mucho más rica que el inglés que les sirve a estos de cortada.

Para Weber (1983), entre las distintas estrategias de relativización del quechua se hallarían las siguientes:

a) Orden opcional entre la cabeza y el modificador (shamushan wamrakuna-ta-pis / wamrakuna shamushan-ta-pis 'incluso los chicos que vinieron').

b) Supresión del marcador oblicuo (yaku-man yayku-shan roopa-wan chakikuykan / yaku-man yayku-shan roopa chakikuykan 'las ropas (con) las cuales se metió al agua están secándose')..$^{15}$

c) Presencia / ausencia de núcleo en la cláusula relativa (hitari-shayki llachapa-ta rikashkaa / hitari-shayki -ta rikashkaa 'he visto las (ropas) que tú tiraste').

d) Extraposición de la frase nominal (mana paykuna-pa mandun-kuna achka-n-chu 'no son muchos los subordinados suyos' $\rightarrow$ paykuna-pa mana kan-n-chu achka-qa mandun-kuna 'los suyos no son muchos subordinados').

e) Presencia / ausencia de núcleo del SN dominante por sustantivación del verbo y supresión (marka-man chayasha:-chaw hamashkaa 'me quedé en la ciudad a la cual llegué; lit.: 'me quedé en mi llegada a la ciudad').

\footnotetext{
${ }^{15}$ Es una conducta supresora muy frecuente. En español hay una tendencia grande a la eliminación superficial de la preposición (equivalente a la marca de caso en quechua): Lo bajó del árbol [en] que estaba.
} 
f) La frase relativa se convierte en un pronombre indefinido (rikay aywa-shayki-ta 'mira adónde vas' / rikay may-pa aywa-shayki-ta-pis 'mira cualquiera que se el sitio adonde vas').

Es evidente que estos aspectos no fueron estudiados por los misioneros gramáticos. Sólo en GH hay algunos comentarios sobre la desaparición de -qa o chayqa y sus efectos:

...esto es lo comun y ordinario y mas proprio con ambas particulas quando no ay impedimento porque ambas son de la forma de los relatiuos, mas a vezes se suele dejar la primera (ca) como en el exemplo del principio de este §. Pi camaricun chayca, sin el (ca) porque con el concurria con la voz de futuro de indicatiuo (camaricunca); mas ya que suframos que se dexe la (ca), mas no se sufre dexar a (chayca) porque como se ha uisto es el mismo relatiuo el que. Si no es alguna vez que le haga la oracion por participio o con (pi) que dizen el que, que es relatiuo (f. 132r)

Hay también algunas aportaciones clásicas a la presencia de elementos periféricos que hacen de frontera del sintagma relativo y cuya movilidad se tiene en cuenta en algún momento, pero los misioneros no profundizan en detalles como los analizados por Weber. La razón que se me ocurre es simple: que todas las ciencias avanzan y no había de dejar de hacerlo la lingüística, pero también que las oraciones de relativo se insertaban en las gramáticas generales y no se redactaban estudios monográficos de sus características por no estar la disciplina oficialmente instituida como tal ni servir a los objetivos próximos ni remotos de los redactores de estos estudios. No obstante, estoy seguro de que en las discusiones lingüísticas diarias de los misioneros, saldrían más de una vez asuntos de esta o similar complejidad. De hecho, aspectos tan complejos como la relación entre las cláusulas relativas y el caso del nombre, aspecto que estudian por ejemplo Lefebvre \& Muysken (1982: § 2.2.2, 2.4 y 3.1.2) es también tratado por los misioneros lingüistas. ${ }^{16}$

Respecto a otros relativos, a los de carácter adverbial resueltos en español mediante donde, cuando, cuanto, como, la bibliografía actual les dedica mucho espacio (Pruñonosa Tomás 1990), sobre todo para distinguir la idiosincrasia de los mismos ya como adverbios, ya como relativos o bien como una categoría híbrida con mezcla de las dos. Pero no tanto la lingüística misionera. No obstante, hay dos excepciones importantes: GH e IRMO. El primero de ellos sugiere lo siguiente, en el apartado de la «oracion relativa por verbos»:

...y desta (chaycca) añado que como es forma de quantos relativos ay, asi su significacion es infinita y no determinada, y a vezes significa como nombre relatiuo y otras como aduerbio con oraciones de tiempo dize entonces, con oraciones de lugar dize alli, o en este lugar y a vezes dize en tal caso, o siendo asi o desa manera, y asi se ha de explicar estirandola a todo lo que pide la primera oracion relatiua (f. 132).

IRMO, que también unifica criterios, reconoce para la cantidad hayca y chicca «que corresponden a tanto, quanto». Por ejemplo: «'quanto hallas, tanto pierdes', ima haycactam

\footnotetext{
${ }^{16}$ Abunda en ello AR, como se vio arriba y presupone GH al ofrecer la declinación de los pronombres relativos (ff. 11v-12v). También lo hace pormenorizadamente Bertonio (1603: 146-166) en su aproximación a los relativos aimaras. No olvidemos dos cosas: que el aimara es una lengua genéticamente muy próxima al quechua (de la familia andina), que dispone también de la partícula -qa, y que Bertonio constituyó con GH, TR y otros jesuitas del momento la llamada escuela de Juli.
} 
tarinqui o Ima chicactam tarinqui, chay chicatam chincachinqui» (f. 41v). Para la cualidad, recurre a hina 'como': «'como hablas, asi obra, ima hinam rimanqui, chay hina / hinatacc ruray». Para la variedad de cosas, IRMO aconseja, en fin, imay mana o haycaymana.

Con ello concluyo asegurando que son muy pocos los aspectos más importantes de la relativización, de los observados hasta ahora por la bibliografía más especializada, que escapan al ojo avizor de los misioneros lingüistas; en efecto, es digno de encomio el cuerpo de doctrina que logran los estudios amerindios de los siglos XVI al XVIII en este prolijo tema del relativo. Es más, se puede generalizar que el cuerpo de doctrina que logra la Gramática Tradicional en todos los campos, desde los orígenes de los estudios misioneros hasta su extinción, no es nada desdeñable, dada la diversidad de lenguas que se incorporan a la disciplina gramatical. Hemos de reivindicar una vez más estos logros frente a los detractores que minimizan los aportes del pasado a los estudios lingüísticos.

\section{Conclusiones}

La oración de relativo falta propiamente en quechua, pero la expresión del adjetivo en forma oracional está presente de muchos modos en esta lengua andina. Así que si no cabe hablar de ello en términos morfológicos, cabe hacerlo y muy en profundidad en términos sintácticos. Eso ha hecho que los lingüistas misioneros hayan aprovechado las enseñanzas gramaticales del latín para proyectar sus argumentos sobre las lenguas indígenas. No obstante, las novedades que aportan éstas obligan a los misioneros a realizar esfuerzos de creatividad que dan frutos importantes, tanto que muchos de los descubrimientos actuales sobre el relativo suponían intuiciones perfectamente identificables como germen de ellos en las reflexiones de los siglos XVI al XVIII.

\section{BIBLIOGRAFÍA}

Alarcos, Emilio (1994): Gramática de la lengua española. Madrid: Espasa Calpe.

Alvares, Manuel (1572): De institvtione grammatica. Lisboa: Ioannes Barrerius.

Anónimo (1586): Arte y Vocavulario en la lengva general del Peru llamada quichua, y en la lengua española. Lima: Antonio Ricardo.

Bertonio, Ludovico (1603): Arte y gramática muy copiosa de la lengua aymara. Roma: Luis Zannetti.

Calvo Pérez, Julio (1993): Pragmática y gramática del quechua cuzqueño. Cuzco: CERA «Bartolomé de las Casas».

Calvo Pérez, Julio (1994): Introducción a la pragmática del español. Madrid: Cátedra.

Calvo Pérez, Julio (en prensa): Nuevo diccionario español-quechua / quechua-español. A publicarse por la Universidad «San Martín de Porres» de Lima (Perú).

Costa, Rachel (1972): «A study of the SQA, NA, Y, and Q nominalizing suffixes in Quechua». En: Papers in Andean Linguistics, 1 (Good Languages University of Wisconsin Anthropology Department, Madison), 29-77.

Gili Gaya, Samuel (1943/197311): Curso superior de sintaxis española. Barcelona: Vox, Bibliograf. González Holguín, Diego (1607/1975): Gramática y arte nueva de la lengva general de todo el Peru, llamada lengua Qquichua, o lengua del Inca. Lima: Francisco del Canto. Edición facsimilar. Vaduz-Georgetown: Cabildo.

Keenan, Edward (1972): «On semantically based grammar». En: Linguistic Inquiry, III, 4: 413-461. 
Keenan, Edward \& Bernard COMRIE (1977): «Noun Phrase Accessibility and Universal Grammar». En: Linguistic Inquiry 8, 63-99.

Lefebvre, Claire \& Pieter Muysken (1982): Relative Clauses in Cuzco Quechua: Interactions between Core and Periphery. Bloomington: Indiana University Linguistics Club (IULC).

López García, Ángel (1994): Gramática del español. I. La oración compuesta. Madrid: Arco Libros. Nebrija (o Lebrija), Antonio de (1492/1981): Gramática de la lengua castellana. Edición de Antonio de Quilis, Madrid: Editora Nacional.

Roxo Mexía y Ocón, Iván (1648): Arte de la lengva general de los indios del Perv. Lima: Jorge López de Herrera.

Santo Thomas, Fray Domingo de (1560/1951): Grammatica o arte de la lengua general de los indios de los reynos del Peru nueuamente compuesta por el Maestro Fray Domingo de S. Thomas. Valladolid: por Francisco Fernández de Córdova. Edición facsimilar de Raúl Porrás Barrenechea. Lima: Imprenta de Santa María.

Snow, Charles (1973): «Nominalization in Ancash Quechua». En: Papers in Andean Linguistics, 2 (Good Languages University of Wisconsin Anthropology Department, Madison), 5-129.

Pruñonosa, Tomás, Manuel (1990): De la cláusula relativa. Los relativos donde y cuando. Valencia: Universidad de Valencia.

Torres Rubio, Diego de (1619/1774): Arte y vocabulario de la lengua quichua general de los Indios de el Peru. Añadida por el P. Juan de Figueredo. Lima: Plazuela de San Christoval. ${ }^{17}$

Weber, David J (1976): Bases para el estudio de la relativización con referencia al quechua. Documento de Trabajo 10. Lima: Instituto Lingüístico de Verano (ILV)-Ministerio de Educación.

Weber, David J (1983): Relativization and Nominalized Clauses in Huallaga (Huanuco) Quechua. Linguistics, vol. 103. Berkeley-Los Ángeles-London: University of California Press.

Wölck, Wolfgang (1987): Pequeño breviario quechua. Lima: Instituto de Estudios Peruanos (IEP).

\section{RELATIVNI ODVISNIK V JEZIKU KEČUA: PRISPEVEK MISIJONARSKIH JEZIKOSLOVCEV}

Brez dvoma je tradicionalna slovnica (med drugim tudi misijonarsko jezikoslovje) bistveno prispevala k splošni jezikoslovni teoriji. Delo misijonarskih jezikoslovcev ne predstavlja zgolj povezave med antično in srednjeveško slovnico na eni strani ter moderno na drugi, temveč je tudi most z 19. stoletjem, ko se moderna slovnica utrdi kot znanost.

V prvem delu članka avtor analizira, kako so misijonarji na območju Andov (pod vplivom Alvaresa in Nebrije) uspeli vključiti teorijo oziralnosti v jezike Kečua. V drugem delu se ocenjuje uporabnost, ki jo misijonarsko jezikoslovje predstavlja za aktualno jezikoslovno teorijo (predvsem slovnico razmerij in generativno slovnico) pri proučevanju oziralnih odvisnikov.

\footnotetext{
${ }^{17}$ Hay edición de 1700. Primera edición (dudosa) de Sevilla o Roma 1603. Segunda edición conocida de Lima: Francisco Lasso, 1619.
} 